Communications in Physics, Vol. 24, No. 4 (2014), pp. 289-311

DOI:10.15625/0868-3166/24/4/5504

\title{
EQUIVALENCE BETWEEN VECTOR MESON DOMINANCE AND UNITARISED CHIRAL PERTURBATION THEORY
}

\author{
LE VIET DUNG \\ Centre de Physique Theorique de l'Ecole Polytechnique, 91128 Palaiseau, France \\ and \\ Institute of Physics, Hanoi, Vietnam \\ TRAN NGUYEN TRUONG \\ National Laboratory for High Energy Physics (KEK), Oho 1-1 Tsukuba, Ibaraki 305, Japan \\ and \\ Centre de Physique Theorique de l'Ecole Polytechnique, 91128 Palaiseau, France \\ E-mail: Tran.Truong@cpht.polytechnique.fr \\ Received 26 November 2014 \\ Accepted for publication 12 December 2014
}

\begin{abstract}
It is explicitly shown that either the approximate solution of the integral equation for the inverse of the pion form facto, $r$ or the result of the Pade approximant method of resumming the one loop Chiral Perturbation Theory $(C P T H)$ are equivalent to the standard vector meson dominance (VMD) models, using the vector meson coupling to two pseudoscalars given by the KSRF relation. Inconsistencies between the one loop CPTH and its unitarised version (or the VMD model) are pointed out. The situation is better for the CPTH calculation of the scalar form factor and the related $S$-wave $\pi \pi$ scattering. The branching ratios of $\tau \rightarrow \pi^{+} \pi^{0} \nu, \tau \rightarrow K \pi v, \tau \rightarrow K^{+} \eta v$ and $\tau \rightarrow K^{+} \bar{K}^{0} v$ using only two inputs as the $\rho$ and $K^{*}$ masses, or the two corresponding rms radii, agree with the experimental data. Using the same number of parameters, the corresponding one loop CPTH calculation cannot explain the $\tau$ data.
\end{abstract}

Keywords: vector meson dominance, unitarity, chiral perturbation theory, unitarised chiral perturbation theory, tau decays calculations, scalar form factor calculation.

\section{INTRODUCTION}

The hypothesis of Vector Meson Dominance (VMD) [1] has proved to be an useful and convenient concept in low energy hadronic physics. It enables us to describe many low energy phenomena, below $1 \mathrm{GeV}$ scale, in a compact and convenient language, although not always correct. An apparently different method was based on the dispersion relation approach which was the main activities of the soft hadronic physics in the fifties. It was soon realised to some authors that the VMD model can conveniently be used to describe the more complicated dispersive approach.

A more recent approach to these problems with a different goal was based on the Chiral Perturbation Theory (CPTH) pioneered by Li and Pagels [2], Lehmann [3], Weinberg [4] and (C)2014 Vietnam Academy of Science and Technology 
Gasser and Leutwyler [5-7]. In the Lehmann's approach, the unitarity relation of the S-matrix was taken into account but not the chiral symmetry breaking effect. In the Weinberg approach, the question of unitarity was ignored giving place to the more systematic approach of the perturbation theory in which the unitarity relation was only satisfied perturbatively, i.e. order by order, but the chiral symmetry breaking effect was taken into account. The latter approach enables us to derive systematic low energy theorems, provided that strong interaction can be treated perturbatively, which is an assumption and has to be demonstrated.

In the late fifties, the problem of the validity of the perturbation theory for strong processes was questioned, but this issue has been ignored in recent numerous studies of Chiral Symmetry using CPTH, either by assuming that the strong interaction involved was not sufficiently strong to invalidate the perturbative approach, or that the CPTH was effectively a low energy power series expansion in momentum which could evade the unitarity constraint.

There are few publications on Chiral Symmetry where chiral symmetry breaking effect and unitarity are simultaneously taken into account. This last approach, combining the CPTH with Unitarity, will be called as the Unitarised Chiral Perturbation Theory (UCPTH). It consists in deriving a similar expression as the one loop CPTH result but is supplemented by using the inverse amplitude, the $N / D$ or the Padé approximant methods in order to satisfy the elastic unitarity relation [3, 8-11]. This procedure enables us to extend the perturbation theory to incorporate low energy resonance or bound state phenomena which the standard CPTH cannot handle. We can, in principle, include the inelastic effects but calculation becomes less simple. Except for a most simple calculation of the inelastic effect, we deal in this article the low energy phenomena where the elastic unitarity relation dominates the calculation.

The elastic unitarity relation for the form factor, which is incorporated in the UCPTH approach, leads to the following two consequences. First, the expression for the form factor is an integral equation of the Muskhelishvilli-Omnès type [12] [13] which should not be solved perturbatively. Second, the phase of the form factor in the low energy region (where the inelastic effect can be neglected), must be the same as that given by the corresponding strong interaction phase shift [21]. The solution of the Muskhelishvilli-Omnès integral equation [12] has a polynomial ambiguity, to be discussed below, but the phase theorem is independent of such an ambiguity. Comparision between theory and experiment should therefore be done with the magnitude and phase of the form factor.

The one loop CPTH calculation for the vector and scalar pion form factor was done a long time ago [5]. A 2 loop CPTH calculation was also recently done $[14,15]$. These papers treated the form factors perturbatively and therefore cannot be used to calculate the $\tau$ decay to 2 pseudoscalars which is dominated by the $\rho$ and $K^{*}$ resonances.

In a recent paper on the study of the isovector form factors [8], it was shown that the question of the unitarity must be respected in order to describe in a simple manner the low energy pion physics with or without resonances. It was explicitly pointed out that two approaches could be used: a) One could use the standard CPTH but after having done the one loop perturbative calculation, one must resum the series by the Padé method in order to satisfy the elastic unitarity relation [3,8-11] (this approach could be regarded as the large number of flavor $N_{f}$ expansion). Other applications of the Padé method in physics have been shown to be successful [16], but we give here the reason why it should be. b) One could write the dispersion relation of the inverse of the form factors or the scattering amplitudes which takes automatically into account of the 
elastic unitarity relation, and then solve approximately the resulting integral equation $[8,17]$. The well-known $N / D$ method could be regarded as an extension of this method but is more flexible.

Both methods yield the same result and give rise to the well-known KSRF relation for the width of the vector meson [18] . It was implicitly shown that these two methods are equivalent to the VMD model $[8,19,20]$ using the $\rho \pi \pi$ coupling as given by the KSRF relation [18]. The present method contains less parameter than the VMD model because the KSRF relation is a direct consequence of our approach and not an added assumption.

In this paper we want to point out the equivalence between the VMD model on one hand, and the Padé, the inverse amplitude and the N/D methods on the other hand, for the calculation of $\pi \pi, K \bar{K}, \pi K$ and $\eta K$ vector form factors. They have the same number of parameters as those used in the one loop CPTH method but yield a much more significantly improved prediction due to the respect of the constraint of the unitarity (by a factor of more than 50 in the $\pi K$ form factors squared) $[8,19,20]$.

We want to improve the inverse amplitude method, by expressing the form factor in terms of the $\mathrm{N}$-function of the N/D method for the corresponding strong amplitude. We briefly give the reason why the $\mathrm{N}$ function used in the vector pion form factor calculation can be approximated by the Weinberg low energy expression.

As mentioned above, the unitarity relation for the form factor would require that its phase must be that of the corresponding strong interaction, if the inelastic effect was unimportant in the physical region of interest [21]. This is the only model independent relation which we can make, apart from the (more fundamental and rigourous) Ward identity on the the non renormalisation of the charge of the pion at the zero momentum transfer. For the vector and scalar pion form factors, because there are no significant inelastic effect below $0.9 \mathrm{GeV}^{2}$, the phase theorem should be valid in this region. Hence the phase of the vector pion form factor should have a phase of $90^{\circ}$ at 770 $\mathrm{MeV}$, the $\rho$ vector meson mass, and similarly, the $\pi \mathrm{K}$ vector form factor has a phase of $90^{\circ}$ at the $K^{*}$ mass. Throughout this article and in the UCPTH approach, we have to rely on this phase theorem to check the validity of our approximation scheme. This point is ignored in the standard CPTH calculations.

As a direct application of the UCPTH method, we show that the branching ratios of $\tau \rightarrow$ VectorMesons $+v$ agree reasonably well with the experimental data using only inputs as the $\rho$ and $K^{*}$ masses, or alternatively, the corresponding rms radii ( which the usual CPTH cannot be used or is wrong, being too low, by a factor of 10 to 50). The branching ratios of $\tau \rightarrow K^{+} \eta v$ and $\tau \rightarrow K^{+} \bar{K}^{0} v$ are however smaller than the observed branching ratio by a factor of 2 . A much better agreement with the data is obtained by introducing the polynomial ambiguity as a simulation of the inelastic effect, e.g. the contribution of the $\omega \pi$ intermediate state in the unitarity relation. By fitting the height of the pion form factor data at the $\rho$ resonance with the first order polynomial, the r.m.s. radius is now in good agreement with the data. Alternatively, using the experimental r.m.s. radius as an input, the magnitude of the pion form factor at the $\rho$ resonance is in a very good agreement with the experimental data.

We also want to show that the inelastic effect of the $K \bar{K}$ states on the pion form factor, the inelastic effect of the $\eta K$ intermediate state on the $\pi K$ form factors are however negligible. A more correct approach, using the coupled two-channel unitarity calculation, will be done in the future. 
From a more fundamental viewpoint, we question the assumption made in the CPTH approach that perturbation theory can be used to study the vector pion form factor which could involve important non-perturbative effects. For this purpose, we want to point out some novel theoretical inconsistencies between the one loop CPTH and its unitarised version UCPTH calculation of the vector pion form factor (or equivalently the VMD model); the same observation is also valid for the P-wave $\pi \pi$ elastic scattering. Our observation on the inconsistency can be extended to the two-loop CPTH calculation, and is conjectured to higher orders. We point out, however, that the problem of unitarity may not be serious in the CPTH calculation of the low energy $\mathrm{I}=0$ scalar form factor or the related elastic $S$-wave $\pi \pi$ scattering.

It is our feeling that, not only because the Breit Wigner form cannot be expanded in a convergent power series of momenta near the $\rho$ resonance, because of this inconsistency, even with higher order loop calculation, CPTH will remain as a incomplete low energy perturbative theory and cannot handle resonance or bound state problems which are a manifestation of the non perturbative effects.

The plan of this paper is organised as follows: In section 2, which is the main part of our paper, we give a detailed calculation of the vector pion form factor by perturbation method and also by the non perturbative inverse amplitude and the N/D methods where the elastic unitarity is satisfied. In section 3 the scalar form factor is calculated also by two methods. We then point out that the use of the perturbation method can be justified here. In section 4 , the equivalence between the UCPTH and the VMD is shown. Section 5 deals with the SU(3) generalisation of the form factors. Section 6 is devoted to the applications of the above calculations to the $\tau$ decays.

\section{CALCULATION OF THE VECTOR PION FORM FACTOR}

a) Theoretical Consideration: One Loop CPTH Result

We begin first by recalling the main features of the vector pion form factor calculation using CPTH $[5,6]$. Let us define the vector pion form factor as:

$$
<\pi^{+}\left(p_{1}\right) \pi^{-}\left(p_{2}\right)\left|V_{\mu}^{3}(0)\right| 0>=i\left(p_{2}-p_{1}\right)_{\mu} V(s)
$$

where $s$ is the momentum transfer squared $s=\left(p_{1}+p_{2}\right)^{2}$ and $V(0)=1$. Using the dimensional regularisation scheme, the one loop CPTH result is [5]:

$$
V^{\text {pert. }}(s)=1+\frac{2 \alpha_{9}^{r}(\mu)}{f_{\pi}^{2}} s+\frac{1}{96 \pi^{2} f_{\pi}^{2}}\left(\left(s-4 m_{\pi}^{2}\right) H_{\pi \pi}(s)-s \log \frac{m_{\pi}^{2}}{\mu^{2}}-\frac{s}{3}\right)
$$

where $s$ is the the momentum transfer, $\alpha_{9}^{r}(\mu)$ is one of the renormalized constants defined by Gasser and Leutwyler [5], $f_{\pi}=93 \mathrm{MeV}$, the experimental value of the pion decay constant, and

$$
H_{\pi \pi}(s)=\left(2-2 \sqrt{\frac{s-4 m_{\pi}^{2}}{s}} \log \frac{\sqrt{s}+\sqrt{s-4 m_{\pi}^{2}}}{2 m_{\pi}}\right)+i \pi \sqrt{\frac{s-4 m_{\pi}^{2}}{s}}
$$

for $s>4 m_{\pi}^{2}$; for other values of $\mathrm{s}, H_{\pi \pi}(s)$ can be obtained by analytic continuation.

We can explicitly introduce the expression for r.m.s radius of the vector form factor in Eq. (2). Using the definition $V^{\prime}(0)=\frac{1}{6} r_{V}^{2}=1 / s_{R_{1}}$ we have:

$$
\frac{1}{s_{R_{1}}}=\frac{2 \alpha_{9}^{r}(\mu)}{f_{\pi}^{2}}-\frac{1}{96 \pi^{2} f_{\pi}^{2}}\left(\log \frac{m_{\pi}^{2}}{\mu^{2}}+1\right)
$$


Using this expression in Eq. (2), we have:

$$
V^{\text {pert. }}(s)=1+\frac{s}{s_{R_{1}}}+\frac{1}{96 \pi^{2} f_{\pi}^{2}}\left(\left(s-4 m_{\pi}^{2}\right) H_{\pi \pi}(s)+\frac{2 s}{3}\right)
$$

It should be noticed that the real part of the sum of the last two terms on the R.H.S of Eq. (4) behaves at low energy as $s^{2}$. In using the r.m.s. radius as an experimental input to renormalise the calculation of $V^{\text {pert. }}(s)$, we can calculate perturbatively the vector form factor to the order of $s^{2}$ (modulo of a logarithm). In the language of the dispersion relation approach, the r.m.s radius of the pion vector form factor is used as the subtraction constant.

\section{b) Non Perturbative Approach: Elastic Unitarity Constraint}

At issue here is whether the perturbative hypothesis used in the CPTH approach justified. We shall discuss this problem later. At the moment, we shall discuss the non perturbative problem by using the integral equation approach. Let us review some fundamental properties of the form factor $\mathrm{V}(\mathrm{s})$. Because of the Ward identity and the analytic property of the form factor, we can write the following disperssion relation for $\mathrm{V}(\mathrm{s})$ :

$$
V(s)=1+\frac{s}{s_{R_{1}}}+\frac{s^{2}}{\pi} \int_{4 m_{\pi}^{2}}^{\infty} \frac{\sigma(z)}{z^{2}(z-s-i \varepsilon)} d z
$$

where an extra subtraction is made for convenience, and the spectral function $\sigma(z)$ must be taken to be real from time reversal invariance.

The Muskhelishvilli-Omnès integral equation [12] is obtained by using the elastic unitarity condition for the spectral function:

$$
V(s)=1+\frac{s}{s_{R_{1}}}+\frac{s^{2}}{\pi} \int_{4 m_{\pi}^{2}}^{\infty} \frac{V(z) e^{-i \delta_{1}(z)} \sin \delta_{1}(z)}{z^{2}(z-s-i \varepsilon)} d z
$$

where $\delta_{1}$ is the $\mathrm{P}$ wave $\pi \pi$ phase shift and we have made 2 subtractions in the dispersion relation for later purpose. Because of the reality condition of the spectral function, in the region where the elastic unitarity relation was valid, the form factor would have the same phase as that of the strong amplitude. This is the content of the Watson phase theorem [21].

The solution for the integral equation (6) is well-known [12] and can also be obtained perturbatively by an infinite iteration of the integral equation:

$$
V(s)=P_{n}(s) \exp \left(\frac{s}{\pi} \int_{4 m_{\pi}^{2}}^{\infty} \frac{\delta_{1}(z)}{z(z-s-i \varepsilon)} d z\right)
$$

where $P_{n}(s)$ is the polynomial ambiguity with real coefficients, normalising to unity at $\mathrm{s}=0$; they could represent, at low energy, uncalculable higher energy inelastic effects. We shall assume at the moment that $P_{n}(s)=1$ in the physical region of interest. As can be seen, the phase theorem due to the unitarity of the S-matrix is automatically satisfied. An extension of this integral equation to include a phenomenological inelastic spectral function was previously considered and its solution is also known [13] (see below).

The solution of the integral equation could have been guessed from the reality condition of spectral function: As mentioned above, from this condition, the phase of the form factor is the phase of the strong amplitude. Because $e^{i \delta}$ is not an analytic function, the only correct solution for 
$V(s)$ is given by Eq. (7). The one loop CPTH solution, Eq. (4), is the once iterated solution of the integral equation Eq. (6): This perturbative result can be obtained by setting V(s) in the integral of Eq. (7) to be unity and $f_{1}^{\text {tree }}(s)=e^{i \delta_{1}(s)} \sin \delta_{1}(s) / \rho(s)=\left(s-4 m_{\pi}^{2}\right) /\left(96 \pi f_{\pi}^{2}\right)$ which is purely real; this result follows from the evaluation of the one loop Feynman graph using the Cutkosky rule for the absorptive part and dispersion relation. The once iterated solution of the MuskhelishvilliOmnès integral equation makes no sense, because the exact solution of this integral equation, as stated above, requires an infinite iteration. This problem was discussed in reference [8].

In order to compute the pion form factor, we can either use the experimental P-wave phase shift $\delta_{1}$ or the calculated $\delta_{1}$ phase shifts (obtained from the construction of a unitarised P-wave amplitude) in Eq. (7). To see the effect of the exact solution of the integral equation, let us observe that the P-wave $\pi \pi$ phase shifts passing through $90^{\circ}$ at the $\rho$ mass, hence we approximate the P-wave phase shifts $\delta$ by $\pi \theta\left(s-s_{\rho}\right)$, the zero width approximation for the $\rho$ resonance; the exact solution obtained from Eq.(7) is therefore $s_{\rho} /\left(s_{\rho}-s\right)$. It is clear from this example that we should develop a power series expansion for the inverse of the form factor, if we wanted to incorporate the low energy property of CPTH and at the same time a non perturbative result to take into account of a possible resonant behavior. This was exactly done in reference [8] because the inverse amplitude has a nice property that the elastic unitarity relation for the $\pi \pi \rightarrow \pi \pi$ partial wave is automatically satisfied.

The non-relativistic limit of this expansion was done a long time ago and is known as the "effective range theory" [23]; it is a momentum power series expansion of $k \cot \delta$ which preserves automatically the unitarity of the S-matrix. This is so because the elastic unitarity of the partial wave enables us to write $f(k)=e^{i \delta} \sin \delta / k=1 / k(1-i \cot \delta)$, where $\mathrm{k}$ is the c.o.m. momentum, hence the power series expansion in $k \cot \delta$ which is the effective range expansion, is an expansion of the inverse of the amplitude. This type of expansion enables us to handle very well the low energy bound state (triplet S-wave) and the resonance (singlet $\mathrm{S}$-wave) of the low energy nucleon-nucleon scattering. The missing part of the Hilbert transform or analyticity is presumably unimportant for a non-relativistic theory. ( Unfortunately, standard explanations of the low energy effective range theory do not emphasize the question of unitarity of the S-matrix which is much more important than the potential shape dependence of the expansion).

As applied to the form factor problem, to the extent that the discontinuity of the left hand cut for the partial wave amplitude can be neglected, or can be treated perturbatively (see below), the pion form factor can straightforwardly be obtained by considering the integral equation for the inverse form factor amplitude [8]. This result is equivalent to resum the perturbation series using the infinite bubble summation of the pion loops.

Let us now carry out our analysis, without making any assumption on the left hand cut structure. Because the form factor has a cut from $4 m_{\pi}^{2}$ to infinity, so does its inverse, apart from the contribution coming from the zeros of the form factor appearing as poles which we assume to be absent here. We shall later give a phenomenological description of these zeros as uncalculable inelastic effect which has not been taken into account here.

The inverse of the form factor $V^{-1}(s)$ satisfies also the same analytic property as the form factor $V(s)$, hence we can write a dispersion relation for it. Assuming that $\mathrm{V}(\mathrm{s})$ does not have a zero or its position is far from the physical region of interest, using the elastic unitarity condition, 
we have:

$$
V^{-1}(s)=1-\frac{s}{s_{R_{1}}}-\frac{s^{2}}{\pi} \int_{4 m_{\pi}^{2}}^{\infty} \frac{V^{*-1}(z) e^{-i \delta_{1}(z)} \sin \delta_{1}(z)}{z^{2}(z-s-i \varepsilon)} d z
$$

From the general property of the analytic function, the partial wave amplitude $f_{1}(s)=$ $e^{i \delta_{1}} \sin \delta_{1} / \rho(s)$ where $\rho(s)=\sqrt{1-4 m_{\pi}^{2} / s}, f_{1}(s)$ can always be written as the product of the 2 cuts, the right hand cut or the unitarity cut and the left hand cut due to the exchanged contributions: $f_{1}(s)=N(s) / D(s)$. Because we use the hypothesis of the elastic unitarity, the right hand cut function $D^{-1}(s)$ has the same phase representation as given by Eq.(7) with the normalisation $D^{-1}(0)=1$ and where we set $P_{n}(s)=1$. Hence we can set $V^{*-1}(s) e^{-i \delta(s)} \sin \delta(s)=\rho(s) N(s)$. Eq. (8) can now be rewritten as:

$$
V^{-1}(s)=1-\frac{s}{s_{R_{1}}}-\frac{s^{2}}{\pi} \int_{4 m_{\pi}^{2}}^{\infty} \frac{\rho(z) N(z)}{z^{2}(z-s-i \varepsilon)} d z
$$

This equation expresses the pion vector form factor in terms of the $\mathrm{N}$ function of the elastic P-wave $\pi \pi$ scattering amplitude instead of the P-wave phase shift $\delta_{1}$ as given by Eq. (7). For the following purpose of calculation, Eq. (9) is more convenient. As mentioned above the function $\mathrm{N}(\mathrm{s})$ involves a dispersion integral of the discontinuity of the partial wave amplitude across the left hand cut involving the effects of the $I=0,1$ and 2 pair of pions exchanged in the crossed channels. Because of the normalisation of the function $\mathrm{D}(0)=1$ and that the Weinberg low energy theorem is operative at low energy for the elastic $\pi \pi$ scattering amplitude, we can write the $\mathrm{N}$ function for the P-wave as:

$$
N(s)=\frac{\left(s-4 m_{\pi}^{2}\right)}{96 \pi f_{\pi}^{2}}\left\{1+96 \pi f_{\pi}^{2} \frac{\left(s-4 m_{\pi}^{2}\right)}{\pi} \int_{0}^{\infty} \frac{\operatorname{Im} N(-z)}{\left(z+4 m_{\pi}^{2}\right)^{2}(z+s)} d z\right\}
$$

Eq. (9) and this equation are still exact, apart from the assumption on the absence of the zeros. It provides an alternative solution to that given by Eq. (7) on how to construct the solution of the form factor problem when the dynamics of the strong interaction is known. We now discuss an approximate scheme for Eq. (9). To calculate the form factor V(s) in Eq. (9) we must know the function $\mathrm{N}(\mathrm{s})$ for $s>4 m_{\pi}^{2}$. In this energy range, the contribution from the $\operatorname{ImN}(\mathrm{s})$ is usually small because the denominator in Eq. (10) never vanishes. Hence we approximate N(s) by the first term on the R.H.S. of Eq. (10), i. e. we neglect the contribution from $\operatorname{ImN}(\mathrm{s})$ or equivalently to use the Weinberg low energy theorem for $\pi \pi$ scattering [24]. The approximation scheme is therefore to start initially with the Weinberg low energy theorem for the partial wave amplitude, then this result can be improved by correcting for the deviation of the Weinberg theorem at higher energy. This procedure is reasonable because we use a twice subtracted dispersion relation in $\mathrm{s}$ which emphasise the low energy contribution at small value of $s$ and hence it is valid to approximate the $\pi \pi$ amplitude by the Weinberg expansion as a first approximation.

The form factor $\mathrm{V}(\mathrm{s})$ can now be written as:

$$
V(s)=\frac{1}{1-s / s_{R_{1}}-\frac{1}{96 \pi^{2} f_{\pi}^{2}}\left\{\left(s-4 m_{\pi}^{2}\right) H_{\pi \pi}(s)+2 s / 3\right\}}
$$

Eq. (11) can also be derived by using the Padé approximant method: 


$$
V^{(0,1)}=\frac{V^{\text {tree }}}{1-\frac{V^{1-\text { loop }}}{V^{\text {tree }}}}
$$

where $V^{\text {tree }}$ refers to the tree amplitude which is equal to unity and $V^{1-l o o p}$ refers to the one loop amplitude, i.e. the last two terms on the R.H.S. of Eq. (4). The Padé approximant method yields the same result as that given by Eq.(11) which satisfies the elastic unitarity relation.

We can justify the use of the Padé method to resum the one loop perturbation series by looking at the imaginary part of the inverse of Eq. (12): it is equal to $-\rho(z) N(z)$ with $N(z)$ given by the tree amplitude and hence containing no left hand cut, or $N(z)=\left(s-4 m_{\pi}^{2}\right) /\left(96 \pi f_{\pi}^{2}\right)$.

The problem of $\pi \pi$ scattering, in the approximation where the contribution of the left hand cut could be neglected, was done a long time ago by Brown and Goble [25]. The more exact calculation with the contribution of the left hand cut and in the chiral limit was done by Lehmann [3] where the real part of the logarithm terms due to the right and left hand cuts cancel each other out in the chiral limit. A more exact one loop perturbation calculation was done by [5] and its unitarised form was given by [9]. In this last work, the calculated P-wave phase shifts agree very well with the experimental data from the $\pi \pi$ threshold to $1 \mathrm{GeV}$. and differs little from those calculated by neglecting the left hand cut contribution.

Similarly, our calculation for the form factor using Eq. (11) differs by at most 1-2\% from the direct calculation of the form factor using Eq. (7), with the P-wave phase shifts given by the unitarised version of the P-wave $\pi \pi$ scattering [9]. Explicit results are given in reference [22]. Hence to the extent that the pion loops are taken into account, the left hand cut contribution to the $\mathrm{N}$-function of the P-wave amplitude can be neglected.

From another point of view, the problem of treating the exchange of $\rho$ and $\sigma$ as particles with their full propagators, in the framework of the generalised linear sigma model, was previously examined in the reference [26]. It was found that, in order to have the validity of the KSRF relation, and treating the problem at the tree level approximation, the effect of $\rho$ and $\sigma$ exchanged in the $t, u$ channels should cancel out approximately, when their masses satisfy the relation $m_{\sigma}=$ $\sqrt{2} m_{\rho}$ which is not in contradiction with the experimental fact. This problem should be further investigated.

The above discussions give a justification for the approximation done by Brown and Goble [25]. In fact Eq. (11) is exactly the inverse of the D-function which is the same as the Omnès function given by Eq. (7).

In this section, we limit ourself to the approximation where the discontinuity of the left hand cut can be neglected in order to make a connection of our calculation scheme with the perturbation series and also with the large number of flavor $N_{f}$ expansion scheme. Objections can be raised against this approximation because of the large violation of the crossing symmetry which we were taught back 30 years ago to be an important property of the field theory. This opinion is certainly correct, but calculations done at that period were without the constraints of chiral symmetry, i.e. without having the low energy theorems which can be used to write subtracted dispersion relations. Subtracted dispersion relations are important because we want to suppress the high energy contributions which are difficult to calculate. With the use of the low energy chiral theorems in dispersion relations, the crossing symmetry difficulties are minimized as the scale of the physical problem at low energy is fixed by the low energy theorems. 


\section{c) Deviation from the Elastic Unitarity Constraint}

As we shall show below, the inelastic contribution in the unitarity relation for the vector pion form factor due to the Kaon loops amounts to a few percent. The situation is quite different when we consider the contribution of the $\omega \pi$ and possibly the $\rho \pi \pi$ channels which are experimentally important. The calculation of this contribution to the pion form factor is quite complicated. We can derive a similar equation to the Muskelishvilli-Omnès equation [13] but the phenomenological application has considerable uncertainties [27].

Instead of the integral equation (6), we have now:

$$
V(s)=1+\frac{s}{s_{R_{1}}}+\frac{s^{2}}{\pi} \int_{4 m_{\pi}^{2}}^{\infty} \frac{V(z) f_{1}^{*}(z)+\sigma_{i}(z)}{z^{2}(z-s-i \varepsilon)} d z
$$

where $f_{1}(s)=\frac{\eta e^{i \delta(s)}-1}{2 i}, \eta$ being the inelastic factor, and $\sigma_{i}(s)$ being the inelastic contribution to the unitarity relation and differing from zero for $\mathrm{s}$ above the inelastic threshold $s_{i}$. The solution for this equation is [13]:

$$
V(s)=\frac{1}{D(s)}\left[1+\frac{1}{\pi} \int_{s_{i}}^{\infty} \frac{2 D(z) \operatorname{Re}\left(\sigma_{i} e^{i \delta}\right) d z}{(1+\eta) e^{-i \delta} z(z-s-i \varepsilon)}\right]
$$

We also have to require that the first derivative of $V(s)$ at $\mathrm{s}=0$ to be $s_{R-1}$.

Below and sufficiently far from $s_{i}$, e.g. the $\omega \pi$ threshold, we can roughly parametrise the contribution of the second term on the R.H.S. of Eq. (14) as a polynomial $P_{n}(s)$ which is real for $s<s_{i}$ and is the polynomial ambiguity in the solution of the Muskhelishvilli-Omnès equation. Because of the normalisation $P_{n}(0)=1$, the introduction of this factor will not influence the Ward identity, but does influence the value of the r.m.s.value of the pion radius. This type of approximation is reliable for energy below the inelastic threshold, but is erronous at the inelastic threshold and also at higher energy.

We shall fit the experimental data below with the simple expression $\left(1+\alpha s / s_{\rho}\right)$, where $\alpha$ is a constant in order to simulate phenomenologically the inelastic effect. This formula was first suggested by Truong [29]. The pion form factor can now be written as:

$$
V(s)=\frac{1+\alpha s / s_{\rho}}{1-s / s_{R_{1}}-\frac{1}{96 \pi^{2} f_{\pi}^{2}}\left\{\left(s-4 m_{\pi}^{2}\right) H_{\pi \pi}(s)+2 s / 3\right\}}
$$

\section{d) Phenomenological Applications}

1) Let us first calculate the form factor without using the phenomenological introduction of the inelastic effect in the unitarity relation. Eq. (11) gives then the expression for the vector pion form factor. Because the vector r.m.s. radius is positive, the vector form factor has a resonant character. Its width satisfies the KSRF relation [18]. The relation between the $\rho$ resonant mass squared $s_{\rho}$ and $s_{R_{1}}$ is:

$$
s_{R_{1}}=\frac{s_{\rho}}{1-\frac{1}{96 \pi^{2} f_{\pi}^{2}}\left\{\left(s_{\rho}-4 m_{\pi}^{2}\right) \operatorname{ReH}_{\pi \pi}\left(s_{\rho}\right)+2 s_{\rho} / 3\right\}}
$$

In Eq.(16), we can either use $s_{R_{1}}$ or $s_{\rho}$ as an input parameter. If the r.m.s. radius was used then one would predict the $\rho$ mass and width which can be seen to satisfy the KSRF relation [18]: 
$\Gamma_{\rho}=\left(s_{\rho}-4 m_{\pi}^{2}\right)^{3 / 2} /\left(96 \pi f_{\pi}^{2}\right)$ where we have neglected the finite width correction [30]. In terms of the $g_{\rho \pi \pi}$ coupling constant, we have:

$$
g_{\rho \pi \pi}^{2}=\frac{s_{\rho}}{2 f_{\pi}^{2}}
$$

where $g_{\rho \pi \pi}$ is defined at $s=s_{\rho}$. Using the experimental value $m_{\rho}=0.77 \mathrm{GeV}$, the uncorrected width of the vector meson $\rho$ is $0.141 \mathrm{GeV}$. Including the finite width correction [30], the $\rho$-width, defined as $\left(\sqrt{s_{\rho}} \Gamma_{\rho}\right)^{-1}=\left.\cot \delta_{1}(s)^{\prime}\right|_{s=s_{\rho}}$, where the sign' denotes the first derivative of $\cot \delta_{1}$ with respect to s. The KSRF relation, with the finite width correction, yields $\Gamma_{\rho}=155 \mathrm{MeV}$ which is very near to the experimental value of $151.5 \pm 1.5 \mathrm{MeV}$.

If one used $s_{\rho}$ as an input parameter, then one would predict the $\rho$ width by the KSRF relation [18], and the r.m.s. radius of the pion. For a number of reasons which will become clear later, we use the $\rho$ mass as an input. The calculated r.m.s. radius is $\left\langle r_{V}^{2}\right\rangle=0.40 \pm 0.01 \mathrm{fm}^{2}$ compared with the experimental value $\left\langle r_{V}^{2}\right\rangle=0.439 \pm 0.008 \mathrm{fm}^{2}$ [31]. The calculated value is therefore too low. This difficulty is related to the fact that the calculated pion form factor at the $\rho$ peak is also too low, as can be seen in Fig. 1 (see below). This result is expected, because we neglected the inelastic contributions in the unitarity relation which does not change the imaginary part of the pion form factor but does contribute to its real part as explained above. A much better agreement with the experimental data, both at the $\rho$ peak and also for the pion r.m.s. radius can be obtained by phenomenologically introducing the inelastic effect.

The phase and modulus squared of the pion form factor calculated by the CPTH, Eq. (4), and by UCPTH, Eqs. $(11,12)$, using $\left\langle r_{V}^{2}\right\rangle=0.40 \pm 0.01 \mathrm{fm}^{2}$ are shown on figures 1 and 2 . Although they both agree with each other at low very momentum transfer, due to the dominance of the r.m.s radius term, the higher energy experiment data are much better represented by the UCPTH calculation than those of the CPTH calculation. Experiences with analytic functions show that a small difference between two analytic functions in one region can be greatly amplified in another region. This explains why the form factors calculated in both CPTH and UCPTH have the same value and radius at $s=0$, but have completely different behavior around the $\rho$ resonant region. The UCPTH calculation has the $\rho$ resonance character while that from the one loop CPTH does not.

The phase theorem of the pion form factor [21] can be used to test whether the unitarity relation is satisfied or not. From Fig. 2, for $s>0.2 \mathrm{GeV}^{2}$ the CPTH phase is clearly much less than those obtained experimental data reflecting that the unitarity relation is badly violated in this approach. The UCPTH pion form factor phase is, on the other hand, in very good agreement with the data from the $2 \pi$ threshold to $1 \mathrm{GeV}^{2}$. This reflects that the UCPTH approach satisfies the unitarity relation in this region. We conclude that the unitarity relation plays a crucial role in the low energy calculation of the pion form factor and also of the $\pi \pi$ scattering. We shall show elsewhere some difficulties of calculating the process $\pi \rightarrow \gamma \gamma^{*}$ encountered in the CPTH approach can also be removed by using the UCPTH method [28].

\section{e) Inadequacy of CPTH calculation for Vector Form Factor.}

Let us now examine Eq. (4). As noted above, the last term in Eq. (4) has a different low s behavior for the real and imaginary parts: at small s, its real part behaves as $s^{2}$ while its imaginary parts, in the chiral limit, behaves as s. This mismatch between the small s behavior of the real and imaginary parts is due to the perturbative approach which results in a violation of the 


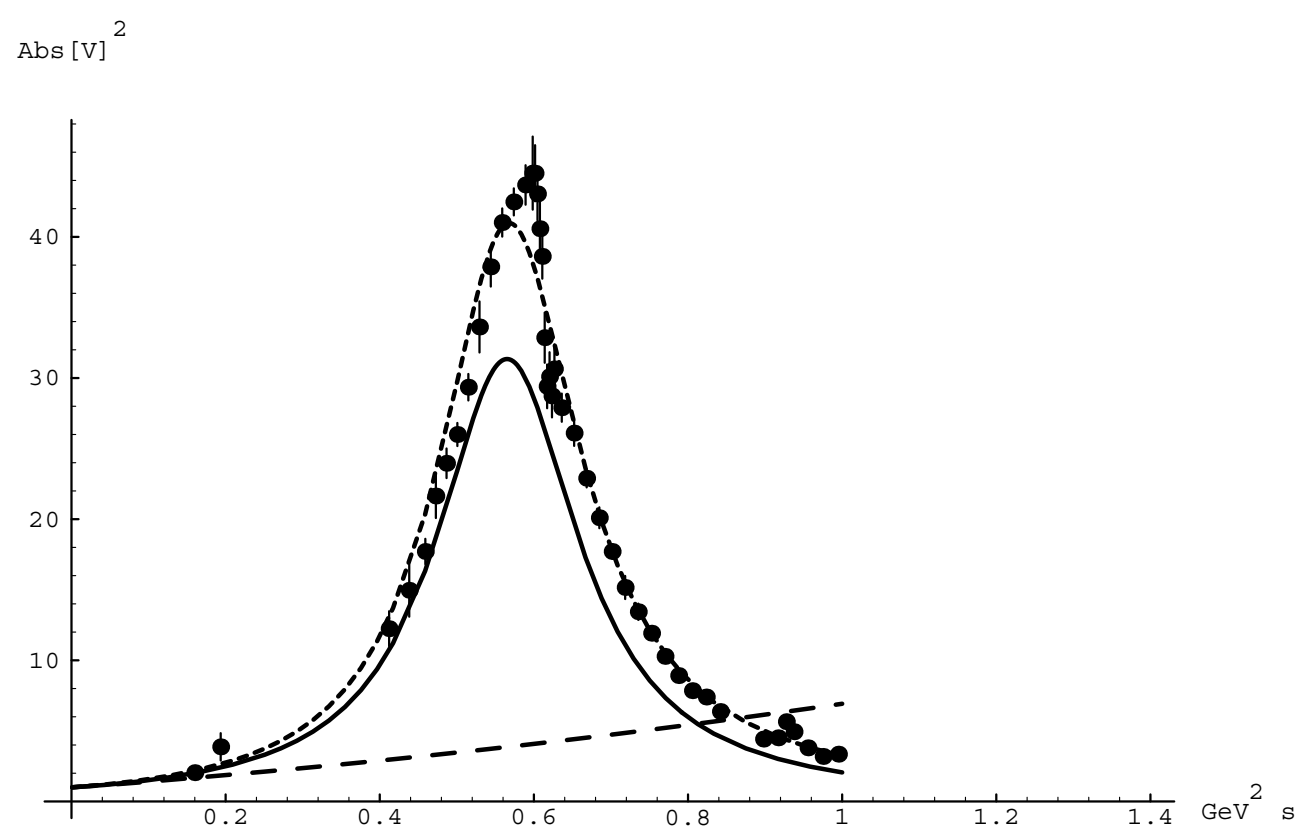

Fig. 1. The square of the modulus of the vector pion form factor $V(s)$ as a function of the energy squared $s$. The experimental data are taken from the review article by D. Morgan and M. Pennington [37]. The solid line curve is the result of the UCPTH, Eq.(11); the dashed curve is the result of the standard one loop CPTH using the same parameter as that of UCPTH Eq.(4); the dotted curve is that obtained by simulating the inelastic $\omega \pi$ contribution [27] by multiplying the UCPTH result with the factor $1+0.15 s / s_{\rho}$.

phase theorem. In order to restore this theorem, even at low energy, the next order term in the imaginary part has to be included in the perturbation calculation as pointed out in ref. [8] and will be discussed later in the scalar form factor calculation. This problem does not exist in UCPTH approach as can be seen from Eq. (11).

From the discussion of the previous paragraph, we are led to the study of the $\mathrm{O}\left(s^{2}\right)$ term in Eq. (4) and that in Eq. (11). Although they are numerically very small at low values of s, because we are interested in extending the region of the validity of the perturbation theory to near the $\rho$ resonant region, they must be taken into account in this energy range. Hence we are led to examine the consistency of the CPTH approach compared with the UCPTH calculation or the VMD model which we shall show later to be equivalent. For this purpose, not only we want to compare the experimental data on the modulus of the pion form factor but we also want to use the phase theorem which is model independent, to compare the calculated phases with those obtained from the experimental data.

From Eq. (11), it is seen that the perturbative approach of Eq. (4) leaves out, at low energy, a term $\left(s / s_{R_{1}}\right)^{2}$ which should be compared with the real part of the last term in Eq. (4) because they are both of the same order in s. The perturbative approach is only justified if the last term is much larger than the former. Let us now define the parameter $r_{V}$, defined as the ratio of the real part of the last term on the R.H.S. of Eq. (4) over $\left(s / s_{R_{1}}\right)^{2}$ : 


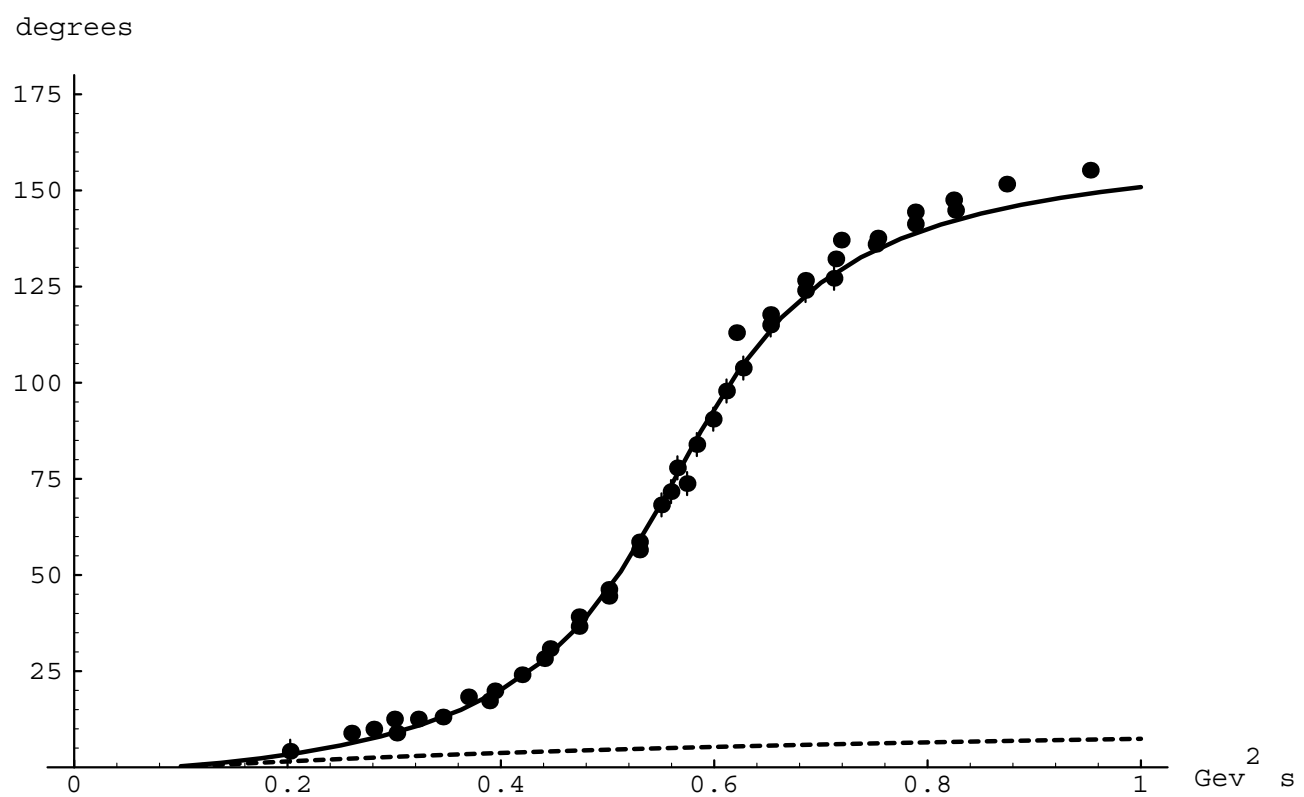

Fig. 2. The phase of the vector pion form factor in degrees as a function of the energy squared $s$. The unitarity relation requires that it has the same phase as that of the strong $\mathrm{P}$ wave $\pi \pi$ scattering. The experimental data for the $\mathrm{P}$-wave phase shift are taken from [37]. The solid curve is the calculated phase of the pion form factor using UCPTH, Eq.(11); the dashed curve is calculated with the CPTH, Eq.(4); the inelastic effect due to the $\omega \pi$ contribution does no affect the phase of the UCPTH form factor below the $\omega \pi$ threshold.

$$
r_{V}=\frac{\frac{1}{96 \pi^{2} f_{\pi}^{2}}\left(\left(s-4 m_{\pi}^{2}\right) R e H_{\pi \pi}(s)+\frac{2 s}{3}\right)}{\left(s / s_{R_{1}}\right)^{2}}
$$

In Fig. 3, the ratio $r_{V}$ is plotted as a function of s. It is seen that, over a wide range of values of $\mathrm{s}$, including small values of $s, r_{V}$ is much less than unity instead of being much larger than unity in order to guarantee the validity of the perturbation theory. As we shall show below the situation is very different in the calculation of the scalar form factor.

We have discussed so far, the CPTH one-loop calculation of the pion form factor. It is not difficult to calculate the two-loop contribution to the vector pion form factor as was previously done numerically by Gaisser and Mei $\beta$ ner [14] and more recently analytically by Colangelo et al. [15]. The main point is that the two loop calculation requires an extra subtraction in the dispersion relation and hence it is necessary to introduce an extra parameter, the second derivative of the pion form factor at the origine. These authors fix this parameter by taking the VMD model which is $\left(s / s_{\rho}\right)^{2}$. What one calculates in the CPTH approach is the contribution of the $s^{3}$ terms, modulo of the logarithm. One can then show the result of the calculated $O\left(s^{3}\right)$ term is still smaller than the $\left(s / s_{\rho}\right)^{3}$ terms coming from the VMD model, although the corresponding $r_{V}$ term is, on the average, a factor of 2-3 larger than the value of $r_{V}$ of the one loop-calculation. 


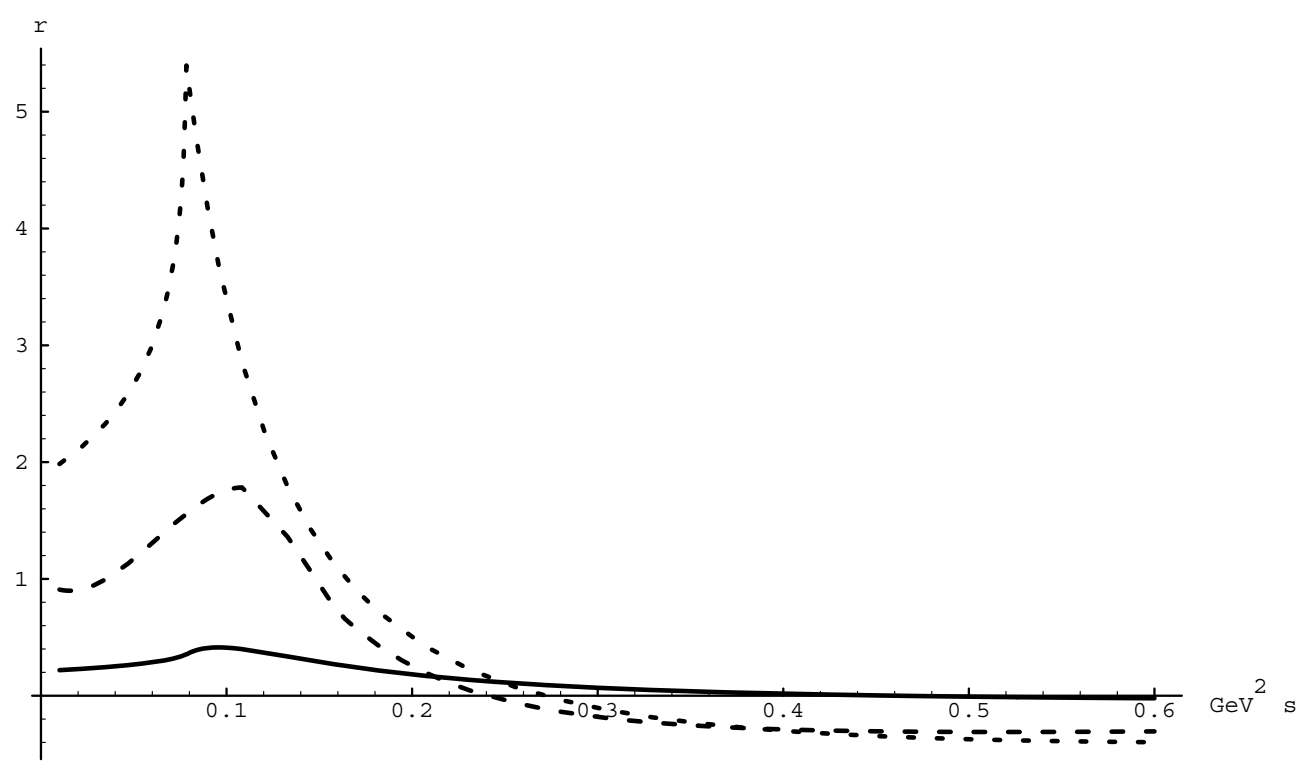

Fig. 3. Test of the convergence of the perturbation expansions. The solid line represents the ratio $r_{V}$ of the one loop vector form factor as a function of the energy squared $s$; the long dashed line represents the corresponding ratio $r_{V}$ for the two loop calculation; the short dashed line represents the ratio $r_{S}$ of the scalar form factor as a function of the energy squared $s$. The convergence of the perturbation series requires that these ratios to be much larger than unity.

Although we have not carried out the calculation of the 3-loop and higher loop amplitudes, we suspect the same difficulty also occurs. This unsatisfactory situation of the 1-loop, 2-loop and possibly higher loop calculations, is due to the use of the perturbation theory to study nonperturbative phenomena. One would have to increase the $\rho$ mass to be larger than $1.5 \mathrm{GeV}$ in order to make accuracy of the vector form factor calculation comparable to the scalar case(see below).

It is so much simpler and more transparent to use Eqs. $(11,12)$ which is even better than the VMD model as a starting point. One then improves it by taking into account of the 2-loop graphs which are neglected in the calculation. A better expression, which takes into account of the inelastic effect yielding a correct r.m.s. radius and the height of the $\rho$ peak Eq. (15), is even better than Eqs. $(11,12)$ for this purpose. If the major goal of physics was to describe experimental data by a few parameters, because CPTH had to rely on the VMD model to get the necessary counter terms, it would become a rather special way of doing physics.

We would like to ask whether by including higher order terms in the CPTH approach that one could generate the Breit-Wigner form. We have a strong doubt about this possibility. Our feeling is based on the experimental fact that there is very little inelastic effect below $1 \mathrm{GeV}$ in the form factor so that higher loop effect cannot be important in the $\rho$ resonance region. 


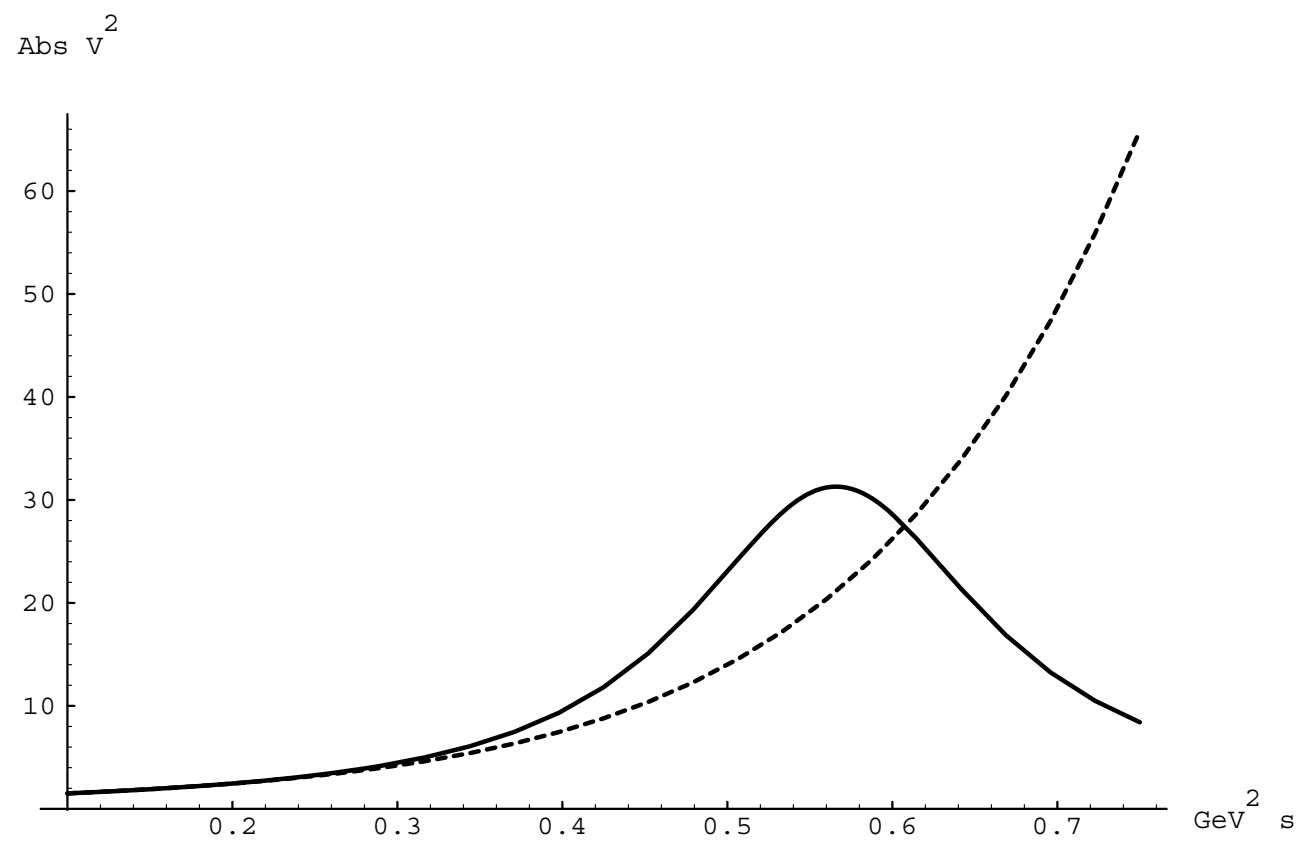

Fig. 4. The modulus of the pion form factor squared calculated by the UCPTH as compared with the sum of the first four terms of the Taylor series expansion of the UCPTH calculation.

In Fig. 4 we plot the modulus of the pion form factor calculated by the UCPTH compared with the sum of the first four terms of the Taylor series expansion of the UCPTH calculation. It is seen that, at low energy, the first four terms are a good approximation for the experimental data but we cannot make the the Breit Wigner form i.e. to make the high energy curve to turn down. This illustrates the problem of the non convergence of the perturbation series for the amplitude; there is, however, no difficulty in generating the Breit Wigner form by expanding the inverse amplitude as a Taylor's series of momenta. Our remark here is a relativistic generalisation of the Bethe's "effective range theory" to handle the resonance and bound state in the two nucleon system [23].

2) We want now to improve our calculation by taking into account of the inelastic effect. As explained above, the simplest parametrisation can be done by setting $P_{n}(s)=1+\alpha s / s_{\rho}$ and is given by Eq. (15).

The good fit to the data can be obtained with $\alpha=0.15$ with $\sqrt{s_{\rho}}=O .77 \mathrm{GeV}$. The vector pion r.m.s. radius squared is now $\left\langle r_{V}^{2}\right\rangle=0.46 \pm 0.01 \mathrm{fm}^{2}$ compared with the experimental value $\left\langle r_{V}^{2}\right\rangle=0.439 \pm .008 \mathrm{fm}^{2}$ [31]. A slightly better fit to the data is obtained with $\alpha=0.13$ but the $\rho$ mass is now changed to $0.773 \mathrm{GeV}$; the vector pion r.m.s. radius squared is now $\left\langle r_{V}^{2}\right\rangle=$ $0.45 \pm 0.01 \mathrm{fm}^{2}$ which is quite good.

Before leaving this section let us write down the dispersion relation for the form factor when the r.m.s. radius is not used as a subtraction constant, but a low energy measured form factor at $s=-s_{0}$ is used as a subtraction constant. Let us consider a measured space-like form factor at $s=-s_{0}$ (the time-like form factor can straightforwardly be written down). Instead of Eq. (6), we 
now have:

$$
\begin{aligned}
V(s)=V(0)+( & \left.V(0)-V\left(-s_{0}\right)\right) \frac{s}{s_{0}} \\
& +\frac{s\left(s+s_{0}\right)}{\pi} \int_{4 m_{\pi}^{2}}^{\infty} \frac{V(z) e^{-i \delta_{1}(z)} \sin \delta_{1}(z)}{z\left(z+s_{0}\right)(z-s-i \varepsilon)} d z
\end{aligned}
$$

The one-loop CPTH result can be obtained perturbatively from this equation by setting in the integrand $V(z)=1$ and $f_{1}^{t r e e}(s)=e^{i \delta_{1}(s)} \sin \delta_{1}(s) / \rho(s)=s /\left(96 \pi f_{\pi}^{2}\right)$. Because we use the input as the measured form factor at $s=-s_{0}$, we cannot discuss the obtained results as an expansion in a power series in $s$.

Let us finally discuss the ghost problem in the Padé, inverse or the N/D methods. Eqs. $(11,12)$ develop a pole at $s_{g}=-3.8 \cdot 10^{5} \mathrm{GeV}^{2}$ which is a ghost and should not be there. It has to be eliminated from these equations by multiplying them with the factor $\left(1-s / s_{g}\right)$ which does not effect the normalisation of the form factor at $s=0$, but change the value of the pion form factor by an amount $s / s_{g}$ which is completely negligible at small $s$. The phase theorem is also unaffected by this factor. We show here that the presence of a ghost due to the unitarisation procedure can be tolerated, as long as it is sufficiently far from the physical region of interest.

\section{CALCULATION OF THE SCALAR FORM FACTOR}

Similarly to the calculation of the vector pion form factor, we can now calculate the isoscalar scalar form factor $\mathrm{S}(\mathrm{s})$ defined as:

$$
<\pi^{a}\left(p_{1}\right) \pi^{b}\left(p_{2}\right)|m(\bar{u} u+\bar{d} d)| 0>=m_{\pi}^{2} \delta^{a b} S(s)
$$

where $s=\left(p_{1}+p_{2}\right)^{2}$. After introducing the scalar r.m.s. radius, using the definition: $S^{\prime}(0)=\frac{1}{6}<$ $r_{S}^{2}>=1 / s_{R_{2}}$ to eliminate the scale dependent $\mu$, the one-loop perturbative result for the scalar form factor is:

$$
S^{\text {pert. }}(s)=1+\frac{s}{s_{R_{2}}}+\frac{1}{16 \pi^{2} f_{\pi}^{2}}\left(\left(s-m_{\pi}^{2} / 2\right) H_{\pi \pi}(s)+\frac{s}{12}\right)
$$

After unitarisation, the UCPTH result for the scalar form factor is:

$$
S(s)=\frac{1}{1-\frac{s}{s_{R_{2}}}-\frac{1}{16 \pi^{2} f_{\pi}^{2}}\left(\left(s-m_{\pi}^{2} / 2\right) H_{\pi \pi}(s)+\frac{s}{12}\right)}
$$

Similarly to the definition of $r_{V}$, we can define $r_{S}$, the ratio of the $O\left(s^{2}\right)$ of the CPTH calculation and $\left(s / s_{R_{2}}\right)^{2}$ :

$$
r_{S}=\frac{\frac{1}{16 \pi^{2} f_{\pi}^{2}}\left(\left(s-m_{\pi}^{2} / 2\right) R e H_{\pi \pi}(s)+\frac{s}{12}\right)}{\left(s / s_{R_{2}}\right)^{2}}
$$

In Fig. (3), the ratio $r_{S}$ is plotted against the energy squared. It is seen that $r_{S}$ is larger than unity, although not much larger on the average; hence, we are assured about the approximate validity of the perturbation theory. The reason for the difference with the vector form factor calculation is due to the coefficient of the chiral logarithm term being larger by a factor 6 in the scalar form factor. This fact explains the partial success of the one loop CPTH calculation in the $\mathrm{S}$-wave $\pi \pi$ scattering ( this was done with the prescription that the phase shift is proportional to 
the real part of the partial wave amplitude in $\pi \pi$ elastic scattering [5]. Had the calculation been done with the definition $\tan \phi=\operatorname{Im} f / \operatorname{Re} f$, the result would be very different).

It should be remarked that in the CPTH approach, the calculated phase of the form factor is not the same as the phase shift $\delta$ because the unitarity relation is not satisfied in the perturbative approach. In fact, if we calculated the phase of the form factor in the CPTH approach, Eq. (21), we would find out that:

$$
\tan \left(\phi_{C P T H}\right)=\frac{\rho(s)\left(s-m_{\pi}^{2} / 2\right)}{16 \pi f_{\pi}^{2}\left\{1+s / s_{R_{2}}+\frac{1}{16 \pi^{2} f_{\pi}^{2}}\left[\left(s-m_{\pi}^{2} / 2\right) \operatorname{ReH}_{\pi \pi}(s)+\frac{s}{12}\right]\right\}}
$$

to be compared with the phase calculated by the UCPTH method, Eq. (22):

$$
\tan \left(\phi_{U C P T H}\right)=\frac{\rho(s)\left(s-m_{\pi}^{2} / 2\right)}{16 \pi f_{\pi}^{2}\left\{1-s / s_{R_{2}}-\frac{1}{16 \pi^{2} f_{\pi}^{2}}\left[\left(s-m_{\pi}^{2} / 2\right) \operatorname{ReH}_{\pi \pi}(s)+\frac{s}{12}\right]\right\}}
$$

In Fig. 5 the dashed curve is the result of the standard one loop CPTH using the same parameter as that of UCPTH Eq. (4). The phases of the scalar form factor calculated by CPTH and UCPTH, $\phi_{C P T H}$ and $\phi_{C P T H}$ are plotted against the energy squared. It is seen the phase of the form factor calculated by CPTH is in a much better agreement with the data than the vector case; the agreement between the UCPTH calculation and the experimental data is, however, better than the phase of the one-loop CPTH calculation. It should be recalled that the phase shift calculated by the CPTH method was done by making the prescription that it is proportional to the real part of the strong S-wave $\pi \pi$ partial wave and agrees well with the experimental data. The difference between the CPTH phase of the form factor and the phase shift is therefore a measure of the violation of the unitarity relation.

It is seen that these two phases are different even at the threshold. Let us compute the limit $s \rightarrow 4 m_{\pi}^{2}$ for $\tan \phi / \rho(s)$. From these two equations and use the definition for the scattering length $a$, the limit $s \rightarrow 4 m_{\pi}^{2}$ of $\tan \phi=(1 / 2) \sqrt{s-4 m_{\pi}^{2}} a$, we get:

$$
a_{C P T H}=\frac{7 m_{\pi}}{32 \pi f_{\pi}^{2}}\left(1+\frac{4 m_{\pi}^{2}}{s_{R_{2}}}+\frac{11 m_{\pi}^{2}}{24 \pi^{2} f_{\pi}^{2}}\right)^{-1}
$$

and

$$
a_{U C P T H}=\frac{7 m_{\pi}}{32 \pi f_{\pi}^{2}}\left(1-\frac{4 m_{\pi}^{2}}{s_{R_{2}}}-\frac{11 m_{\pi}^{2}}{24 \pi^{2} f_{\pi}^{2}}\right)^{-1}
$$

For $s_{R_{2}}=0.56 \mathrm{GeV}^{2}$ which agrees with the experimental scalar radius $\left.<r_{s}^{2}\right\rangle=0.41 \mathrm{fm}^{2}$, we have: $a_{C P T H}=0.123 m_{\pi}^{-1}$ and $a_{U C P T H}=0.23 m_{\pi}^{-1}$. These values are to be compared with the direct CPTH calculation of $\pi \pi$ scattering $a=0.20 m_{\pi}^{-1}$ [5] and the same process using UCPTH, $a=(0.26 \pm 0.02) m_{\pi}^{-1}[9]$.

It is clear that the phase theorem for the form factor is violated in the CPTH method. How can we rectify this situation for CPTH method? As we mentioned above, there is a mismatch between the real and imaginary part of our calculation. In the perturbative calculation, the real part of the form factor is calculated to the second order in $\mathrm{s}$ while its imaginary part is only calculated to the first order in s. In order to compute correctly, in the CPTH approach, the phase $\phi$ of the form factor given by $\tan \phi=\operatorname{ImS} / \operatorname{ReS}$, we must also calculate the imaginary part of the 


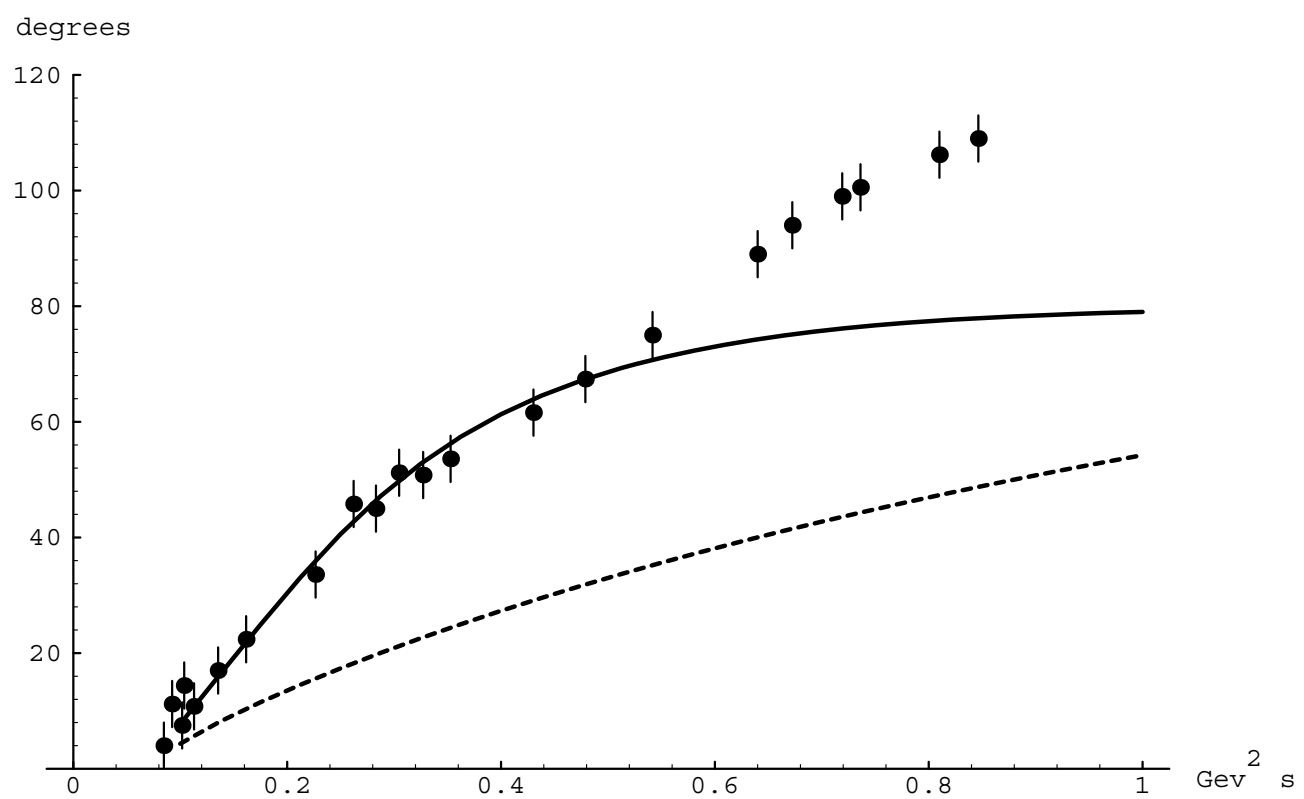

Fig. 5. The phases of the scalar pion form factor, in degrees, as a function of the energy squared $s$. The experimental data are taken from the review article by D. Morgan and M. Pennington [37]. The solid line curve is the result of the UCPTH, Eq.(25); the dashed curve is the result of the standard one loop CPTH using the same parameter as that of UCPTH, Eq.(24).

form factor to the second order in s, i.e. the two loop order for its imaginary part. More precisely, the perturbative unitarity relation for $\operatorname{ImS}$ including the two loop contribution is:

$$
\operatorname{ImS}(s)=\rho(s)\left\{S_{1}(s) f_{1}(s)^{*}+S_{2}(s) f_{1}^{*}(s)+S_{1}(s) f_{2}^{*}(s)\right\}
$$

where $S_{1}$ and $S_{2}$ are, respectively, the first and the remaining terms on the right-hand side of Eq. (21); they represent the tree and one loop amplitudes. Similarly $f_{1}$ and $f_{2}$ are the correponding elastic $\pi \pi$ scattering amplitudes. The calculated scattering length is now :

$$
a=\frac{7 m_{\pi}}{32 \pi f_{\pi}^{2}}\left\{1+\frac{\operatorname{Re} f_{2}\left(4 m_{\pi}^{2}\right)}{f_{1}\left(4 m_{\pi}^{2}\right)}\left(1+\frac{4 m_{\pi}^{2}}{s_{R_{2}}}+\frac{11 m_{\pi}^{2}}{24 \pi^{2} f_{\pi}^{2}}\right)^{-1}\right\}
$$

Using the value $\operatorname{Re} f_{2}\left(4 m_{\pi}^{2}\right) / \operatorname{Re} f_{1}\left(4 m_{\pi}^{2}\right) \simeq 0.25$ from the one loop calculation for the $\mathrm{I}=0$ $\pi \pi$ scattering amplitude [5,9], this Eq. yields $a=0.195 m_{\pi}^{-1}$. This value is very close to the scattering length $a=0.20 m_{\pi}^{-1}$ obtained by CPTH method [5] for the elastic $\pi \pi$ scattering using the prescription that the phase shift $\delta$ is equal to real part of the calculated partial wave.

We see that by correcting for the low energy mismatch of the real and the imaginary parts we can get a reasonable agreement with the phase theorem. A small discrepancy between the new value of the scattering length $0.195 m_{\pi}^{-1}$, with that obtained from UCPTH approach $0.23 m_{\pi}^{-1}$, is due to the fact that $r_{S}$ given by Eq. (23) is not much larger than unity. 
For a more complete 2 loop calculation of the scalar pion form factor, see the recent work of Gasser and Mei $\beta$ ner and Dognohue et. al. [14], [32]. It should be remarked that the sum of the last 2 terms on the R.H.S. of Eq. (III) is not necessarily real, one must take their real part in the calculation.

Eq. (22) develops a pole at $s_{g}=-2.7 \mathrm{GeV}^{2}$ which is a ghost and has to be removed by multiplying the R.H.S. of this Eq. a factor $\left(1-s / s_{g}\right)$ which does not affect the phase theorem, but does change the modulus of the form factor. Unlike the vector pion form factor, this ghost is near to the physical region and has to be taken into account. At $s=m_{K}^{2}$, where $m_{K}$ is the Kaon mass, the correction enhances the modulus of the scalar pion form factor by $10 \%$.

To end this section let us remark that in a recent study, the role of the left hand cut for the scalar form factor has recently been studied in details by Boglione and Pennington [33] in connection with the criticism of the recent work of Dobado and Pelaez [17]. They found that this method is an unreliable way of determining the chiral parameters but a good fit to the data can be done with an appropriate adjustment of the subtraction constant. We agree with this statement, but would like to point out that the situation for the vector pion form factor is different as discussed above.

\section{UCPTH AND VECTOR MESON DOMINANCE}

In the zero width approximation and at the tree level, the vector pion form factor can be written as:

$$
V^{0}(s)=\frac{f_{\rho}^{0} g_{\rho \pi \pi}^{0}}{s_{\rho}-s}
$$

where $f_{\rho}^{0}$ and $g_{\rho \pi \pi}^{0}$ are, respectively, the photon- $\rho$ coupling (multiplying with e) and the strong $\rho \pi \pi$ coupling with $f_{\rho}^{0} g_{\rho \pi \pi}^{0} / m_{\rho}^{2}=1$. At the tree level, these coupling constants should be independent of s, but loop corrections to the 2 and 3 point functions can introduce the s dependence. We now want to make a finite width correction to this Eq. by introducing the self energy correction for the $\rho$ propagator. Summing the geometric series for the $\rho$ self energy and performing the mass and wave-function renormalisations, we have:

$$
V(s)=\frac{f_{\rho}(s) g_{\rho \pi \pi}(s)}{s_{\rho}-s-\pi_{\rho}(s)}
$$

with

$$
\operatorname{Re} \pi_{\rho}(s)=\frac{\left(s-s_{\rho}\right)^{2}}{\pi} P \int_{4 m_{\pi}^{2}}^{\infty} \frac{\operatorname{Im} \pi_{\rho}(z)-\operatorname{Im} \pi_{\rho}\left(s_{\rho}\right)-\left(z-s_{\rho}\right) \operatorname{Im} \pi_{\rho}^{\prime}\left(s_{\rho}\right)}{\left(z-s_{\rho}\right)^{2}(z-s)} d z
$$

and

$$
\operatorname{Im} \pi_{\rho}(s)=\frac{1}{48 \pi} \frac{\left(s-4 m_{\pi}^{2}\right)^{3 / 2}}{\sqrt{s}} g_{\rho \pi \pi}^{2}(s)
$$

where P stands for the principal part integration. The R.H.S of Eq. (32) could be straightforwardly expressed in terms of the function $H_{\pi \pi}(s)$ and its derivatives, if $g_{\rho \pi \pi}(s)$ was a constant. In the usual VMD model, $g_{\rho \pi \pi}(s)$ and $f_{\rho}(s)$ are constants hence $g_{\rho \pi \pi} f_{\rho} /\left(s_{\rho}-\pi(0)\right)=1$. If we neglected the finite width correction, we would have $s_{R_{1}}=s_{\rho}$ and hence the KSRF relation becomes $g_{\rho \pi \pi}^{2}=$ $s_{R_{1}} /\left(2 f_{\pi}^{2}\right)$. Let us now use a theorem stating that two real analytic functions, having the same discontinuity, can only differ by a real polynomial. By comparing Eqs. (32), (33) with Eq. (11) 
we see that they have the same imaginary part and satisfy the same boundary condition and high energy behavior, hence they must be identical. In sum, we have the equivalence between the UCPTH and the VMD.

We could improve the Vector Meson Dominance result by introducing the inelastic effect in the vertex correction, e.g. the $\rho \rightarrow \omega \pi$ to $2 \pi$ state. This correction can be phenomenological represented by a real polynomial in $\mathrm{s}$, and in order to fit the experimental r.m.s radius, we could choose this polynomial as $\left(1+0.15 s / s_{\rho}\right)$ which yields the same result as the calculation of the pion form factor with the inelastic contribution, Eq. (15).

\section{GENERALISATION TO SU(3)}

It is straightforward to generalise the CPTH and UCPTH to SU(3). We have to calculate in addition, the $K \bar{K}$ contribution to the pion form factor. Similarly we calculate the $\pi K$ vector form factors with the $\pi K$ and $\eta K$ contributions and also the $K \bar{K}$ and $\eta K$ form factors. Because the scalar $\eta K$ form factor is negligible due to the small difference of the $\mathrm{K}$ and $\eta$ masses; the scalar $\pi K$ scalar form factor is previously well calculated, we refer the readers to the original calculation [19]. Because we later want to calculate the $\tau$ decay rate, let us now calculate the charge current (isovector) matrix element which we denote now by $V_{i j}$, where $\mathrm{i}$ and $\mathrm{j}$ denote the pseudoscalars. The normalisation of $V_{i j}$ at zero momentum transfer is given by the AdemolloGatto theorem [34]. Using UCPTH, the $\pi^{+} \pi^{0}$ form factor is given by:

$$
V_{\pi^{+} \pi^{0}}(s)=\sqrt{2}\left\{1-\frac{s}{s_{R_{1}}}-\frac{1}{96 \pi^{2} f_{\pi}^{2}}\left[\left(s-4 m_{\pi}^{2}\right) H_{\pi \pi}(s)+\frac{2 s}{3}+\frac{1}{2}\left(\left(s-4 m_{K}^{2}\right) H_{K \bar{K}}(s)+\frac{2 s}{3}\right)\right]\right\}^{-1}
$$

where the subscripts in the function $\mathrm{H}$ refer to the intermediate states defining this function. Similarly we calculate the $K^{+} K^{0}$ isovector vector form factor:

$$
V_{K^{+} \bar{K}^{0}}(s)=\left\{1-\frac{s}{s_{R_{1}}}-\frac{1}{96 \pi^{2} f_{\pi}^{2}}\left[\frac{1}{2}\left(\left(s-4 m_{\pi}^{2}\right) H_{\pi \pi}(s)+\frac{2 s}{3}\right)+\left(\left(s-4 m_{K}^{2}\right) H_{K \bar{K}}(s)+\frac{2 s}{3}\right)\right]\right\}^{-1}
$$

It should be noticed at this point that in the VMD calculation, the $V_{\pi^{+} \pi^{0}}(s)$ and $V_{K^{+} \bar{K}^{0}}(s)$ should be equal and is given by the expression for $V_{\pi^{+}} \pi^{0}(s)$.

The problem of $K \pi$ form factor is discussed in some details in reference [19]. We quote their results on the calculation of the contribution of $K \pi$ intermediate state and add to it the contribution of the $K \eta$ intermediate state (or loop). To simplify our writing, let us define the following function for the unequal mass case:

$$
\bar{H}_{i j}\left(s, m_{i}^{2}, m_{j}^{2}\right)=s^{2} \int_{\left(m_{i}+m_{j}\right)^{2}}^{\infty} \frac{\lambda^{3 / 2}\left(z, m_{i}^{2}, m_{j}^{2}\right) / z^{3 / 2}}{z^{2}(z-s-i \varepsilon)} d z
$$

where $\lambda\left(s, m_{i}^{2}, m_{j}^{2}\right)=\left(s-\left(m_{i}+m_{j}\right)^{2}\right)\left(s-\left(m_{i}-m_{j}\right)^{2}\right)$. The function $\bar{H}_{i j}\left(s, m_{i}^{2}, m_{j}^{2}\right)$ can be expressed in terms of the logarithm function as was done in the references $[6,19]$. In term of this function, we can write down now the expression for the $K^{0} \pi^{+}$and $K^{+} \pi^{0}$ vector form factors:

$$
\begin{gathered}
V_{K^{0} \pi^{+}}(s)=\left\{1-\frac{s}{s_{R_{3}}}-\frac{1}{128 \pi^{2} f_{\pi}^{2}}\left(\bar{H}_{\pi K}\left(s, m_{\pi}^{2}, m_{K}^{2}\right)+\bar{H}_{\eta K}\left(s, m_{\eta}^{2}, m_{K}^{2}\right)\right)\right\}^{-1} \\
V_{K^{+} \pi^{0}}(s)=\frac{1}{\sqrt{2}}\left\{1-\frac{s}{s_{R_{3}}}-\frac{1}{128 \pi^{2} f_{\pi}^{2}}\left(\bar{H}_{\pi K}\left(s, m_{\pi}^{2}, m_{K}^{2}\right)+\bar{H}_{\eta K}\left(s, m_{\eta}^{2}, m_{K}^{2}\right)\right)\right\}^{-1}
\end{gathered}
$$


and the $K \eta$ form factor is given by:

$$
V_{K^{+} \eta}(s)=\sqrt{\frac{3}{2}}\left\{1-\frac{s}{s_{R_{3}}}-\frac{1}{128 \pi^{2} f_{\pi}^{2}}\left(\bar{H}_{\pi K}\left(s, m_{\pi}^{2}, m_{K}^{2}\right)+\bar{H}_{\eta K}\left(s, m_{\eta}^{2}, m_{K}^{2}\right)\right)\right\}^{-1}
$$

Strictly speaking, all above equations are derived not by the inverse amplitude method but rather by the Padé approximant method. It should also be mentioned that the $K^{+} \bar{K}^{0}$ and the $K^{+} \eta$ form factors cannot be calculated at their threshold values by CPTH technique because their thresholds are above the resonant masses.

The vector pion r.m.s. radius squared is calculated to be $\left\langle r_{V}^{2}\right\rangle=0.40 \pm 0.01 \mathrm{fm}^{2}$ compared with the experimental value $\left\langle r_{V}^{2}\right\rangle=0.439 \pm .008 \mathrm{fm}^{2}$ [31]. The vector $K \pi$ r.m.s. radius squared is calculated to be $\left\langle r_{V}^{2}\right\rangle=0.27 \pm 0.008 \mathrm{fm}^{2}$ compared with the experimental value $\left.<r_{V}^{2}\right\rangle=0.34 \pm .03 \mathrm{fm}^{2}[36]$. The agreement between the theory and the experimental data is not satisfactory (see below).

Including the inelastic effect due to the $K^{+} \bar{K}^{0}$ intermediate state in the pion form factor calculation, changes little the numerical result. In a more elaborate calculation [22] where the $\pi \pi$ phase shifts were calculated first which includes the left hand cut contribution to the partial wave strong $\pi \pi$ amplitudes and then compute the vector pion form factor using Eq. (7), there was very little change from the result of the present calculation. The contribution of the $\omega \pi$ inelastic effect in the unitarity equation for the form factor is, however important [27].

The effect of the left hand cut in the P-wave elastic amplitude is, however, more important in the Vector $K \pi$ form factor calculation $[19,22]$.

\section{APPLICATION TO TAU DECAYS}

Obviously CPTH cannot be used for calculations in $\tau$ decays except for a very small region of the phase space where the one-loop calculation is valid [15]. In this region of the phase space, the CPTH results are the same as those obtained by the UCPTH, but the latter method has a much wider range of validity. Because UCPTH can handle resonance, we can use this method to calculate the $\tau$ hadronic decays. Let us now calculate the decay of the lepton $\tau$ into 2 pseudoscalars $\mathrm{i}$ and $\mathrm{j}$. Let us define the ratio $R_{i j}$ of the rate of $\tau \rightarrow P_{i} P_{j} v$ to that of $\tau \rightarrow e v \bar{v}$. Taking only into account of the vector form factor contribution, we have:

$$
R_{i j}=\frac{1}{4 m_{\tau}^{2}}\left(\begin{array}{c}
\cos ^{2} \theta_{c} \\
\sin ^{2} \theta_{c}
\end{array}\right) \int_{s_{t}}^{M^{2}} \frac{\lambda\left(s, m_{i}^{2}, m_{j}^{2}\right)^{3 / 2}}{s^{3}}\left(1+2 s / m_{\tau}^{2}\right)\left(1-s / m_{\tau}^{2}\right)^{2}\left|V_{i j}(s)\right|^{2} d s
$$

where $\theta_{c}$ is the Cabbibo angle with $\sin ^{2} \theta_{c}=(0.222)^{2}, s_{t}$ is the square of the threshold energy and $\mathrm{M}$ is the $\tau$ lepton mass. Let us use the following notation $R_{i j}(a b)$ to denote the ratio $\mathrm{R}$ defined in Eq. (40) with intermediate states a and b and $R_{\pi K}$ means the sum of the decay rate into $\pi^{+} K^{0}$ and $\pi^{0} K^{+}$. Using Eqs. (34-39), we have:

$$
\begin{array}{rcl}
R_{\pi^{+} \pi^{0}}(\pi \pi)=1.05 & R_{\pi^{+} \pi^{0}}(\pi \pi, K \bar{K})=1.03 & \left.R_{\pi^{+} \pi^{0}}\right|_{\exp }=1.38 \pm .02 \\
R_{K^{+} \bar{K}^{0}}(\pi \pi)=0.0042 & R_{K^{+} \bar{K}^{0}}(\pi \pi, K \bar{K})=0.0031 & \left.R_{K^{+} \bar{K}^{0}}\right|_{\exp }=.0075 \pm .002 \\
R_{\pi K}(\pi K)=0.050 & R_{\pi K}(\pi K, \eta K)=0.048 & \left.R_{\pi K}\right|_{\exp }=0.065 \pm .008 \\
R_{\eta K}(\pi K)=5.1 .10^{-4} & R_{\eta K}(\pi K, \eta K)=4.1 .10^{-4} & \left.R_{\eta K}\right|_{\exp }=(1.3 \pm .04) 10^{-3}
\end{array}
$$


The experimental data are taken from the recent paper of the CLEO group [35] and the Particle Data Group [36].

It is seen that our calculation for the $\rho$ and $K^{*}$ decays are too small by $35 \%$ compared with the experimental rates. This is expected because we neglect the inelastic contribution and essentially use the threshold parameters, the r.m.s. radii as inputs to extrapolate to the resonance region which is far away.

Similarly the $K^{+} \bar{K}^{0}$ and $K^{+} \eta$ decays are too low by a factor of 2. (The finite width correction is very small for the $K^{*}$ : the KSRF relation gives $\Gamma_{K^{*}}=55.2 \mathrm{MeV}$ and the finite width correction with $\pi K$ loop gives $55.6 \mathrm{MeV}$ and with $\pi K$ and $\eta K$ loops give $53.4 \mathrm{MeV}$ ). The scalar form factor contribution to the above decay rates are small. Their maximum contribution is in $R_{\pi K}$ which amounts only to $3-4 \%$ of the calculated rate [19].

If we are willing to introduce more parameters in our calculation in order to fit the data on the top of the resonances and want only to predict the r.m.s. radii and the inelastic form factors $K^{+} \bar{K}^{0}$ and $K^{+} \eta$, we can certainly do a better job. This can be done by using the polynomial in Eq. (7) as explained above.

Because on the top of the $\rho$ resonance the peak value of the form factor is too low by $30 \%$, we can set $P_{n}(s)=\left(1+0.15 s / s_{\rho}\right)$ in order to have the correct peak value, and hence we multiply the right hand side of Eqs. (34-39) by this factor.

The vector pion r.m.s. radius squared is now $\left\langle r_{V}^{2}\right\rangle=0.46 \pm 0.01 \mathrm{fm}^{2}$ compared with the experimental value $\left\langle r_{V}^{2}\right\rangle=0.439 \pm .008 \mathrm{fm}^{2}$ [31]. The vector $K \pi$ r.m.s. radius squared is now $\left.<r_{V}^{2}\right\rangle=0.31 \pm 0.008 \mathrm{fm}^{2}$ compared with the experimental value $\left\langle r_{V}^{2}\right\rangle=0.34 \pm .03 \mathrm{fm}^{2}$ [36] There is now a good agreement with the data.

The $\tau$ decay rates are now:

$$
\begin{array}{rcl}
R_{\pi^{+} \pi^{0}}(\pi \pi)=1.41 & R_{\pi^{+} \pi^{0}}(\pi \pi, K \bar{K})=1.38 & \left.R_{\pi^{+} \pi^{0}}\right|_{\exp }=1.38 \pm .02 \\
R_{K^{+} \bar{K}^{0}}(\pi \pi)=0.067 & R_{K^{+} \bar{K}^{0}}(\pi \pi, K \bar{K})=0.064 & \left.R_{K^{+} \bar{K}^{0}}\right|_{\exp }=.075 \pm .02 \\
R_{\pi K}(\pi K)=0.067 & R_{\pi K}(\pi K, \eta K)=0.064 & \left.R_{\pi K}\right|_{\exp }=0.065 \pm .008 \\
R_{\eta K}(\pi K)=8.9 .10^{-4} & R_{\eta K}(\pi K, \eta K)=7.2 .10^{-4} & \left.R_{\eta K}\right|_{\exp }=(1.3 \pm .04) 10^{-3}
\end{array}
$$

It is seen that the agreement with the data is much better which is not surprising because we do not have such a long range of energy to extrapolate, from the resonant energy to the $\eta K$ and $K \bar{K}$ energy available in the $\tau$ decay. These results are changed slightly when we change the $\rho$ mass to $0.773 \mathrm{GeV}$ and the correction factor to $\left(1+0.13 s / s_{\rho}\right)$.

\section{CONCLUSION}

We have presented here the study of the form factor problem using the UCPTH approach. The main feature of this method is that, at low energy, it coincides with that derives from the CPTH method. Because of the unitarity constraint, we are forced to rewrite the CPTH results as an infinite geometric series using either the inverse amplitude method or the Padé method. The former one is more general than the latter. In the simplest approximation to the one loop CPTH, both method yields the same result. They enable us to extend the analysis of CPTH to the resonant region and beyond without introducing more parameters. 
On the top of the $\rho$ and $K^{*}$ masses, our calculation on the magnitudes of the form factors are too low and only accurate to $15 \%$ in the amplitudes. The $\rho$ width is correctly predicted within a few percents, while that of the $K^{*}$ is $10 \%$ too high compared with the data. The r.m.s. radii are in agreement with the data. Extending our calculation to above $1 \mathrm{GeV}$, then the accuracy becomes worse. The $\tau$ to $\rho$ and $K^{*}$ decay branching ratios are accurate to about $35 \%$ but the $\tau$ to $K^{+} \bar{K}^{0}$ and $K^{+} \eta$ are too low by a factor of 2 .

In order to improve our predictions, we modify our UCPTH results which are obtained based on the assumption of the elastic unitarity, by incorporating the inelastic effect through the phenomenological polynomial ambiguity of the Omnès function. Mutiplying the UCPTH results on the form factors by the factor $\left(1+0.15 s / s_{\rho}\right)$ the leptonic widths of the $\rho$ and $K^{*}$ are in agreement with the data. The prediction of the branching ratios $\tau \rightarrow K^{+} \bar{K}^{0} v$ and $K^{+} \eta v$ are also in agreement with the data. The corresponding rms radii are also in agreement with the data.

\section{ACKNOWLEDGEMENT}

It is a pleasure for one of us (T. N. Truong) to thank the hospitality of the Physics Department at the National High Energy Physics Laboratory K.E.K and in particular Prof. Y. Shimizu for hospitality. He also would like to thank the Centre National de Recherche Scientifique for granting a leave of absence to visit Japan, the Hanoi National University, Hochiminh National University and Hue University where part of this work was carried out. (L. V. Dung) wants to thank the Centre de Physique Theorique de l' Ecole Polytechnique for a fellowship and hospitality. We both thank to L. Beldjoudi for having participated in the intial stage of this work.

\section{REFERENCES}

[1] J. J. Sakurai, Ann. Phys. (N. Y.) 11 (1960) 1; Y. Nambu and J. J. Sakurai, Phys. Rev. Lett. 8 (1962) 79, 191 E;

M. Gell-Mann, D. Sharp and W. Wagner, Phys. Rev. Lett. 8 (1962) 261;

For recent development on this subject, see the review article of M. Bando, T. Kugo and K. Yamawaki, Phys. Rep. 164 (1988) 217.

[2] L. F. Li and H. Pagels, Phys. Rev. Lett. 26 (1971) 1204; 27 (1971) 1089.

[3] H. Lehmann, Phys. Lett. B 41 (1972) 529.

[4] S. Weinberg, Physica 96 A (1979) 327.

[5] J. Gasser and H. Leutwyler, Ann. Phys. (N.Y.) 158 (1984) 142.

[6] J. Gasser and H. Leutwyler, Nucl. Phys. B 250 (1985) 465 ibid. B250 (1985) 539.

[7] For a recent review, see J. F. Donoghue, E. Golowich and B. R. Holstein, ” Dynamics of the Standard Model”, pub. Cambridge 1994.

[8] T. N. Truong, Phys. Rev. Lett. 61 (1988) 2526.

[9] A. Dobado, M.J. Herrero and T. N. Truong, Phys. Lett. B 235 (1990) 129, 134.

[10] T. N. Truong, Phys. Rev. Lett. 67 (1991) 2260.

[11] K. S. Jhung and R. S. Willey, Phys. Rev. D 9 (1974) 3132.

[12] N. I. Muskhelishvili, Singular integral equations ( Noordhoff, Groningen, 1953); R. Omnès, Nuovo Cimento 8 (1958) 316.

[13] T. N. Pham and T. N. Truong, Phys. Rev. D 16 (1977) 896. O. Babelon, J. L. Basdevant, D. Caillerie and G. Mennessier, Nucl. Phys. B113 (1976) 402.

[14] J. Gasser and Ulf-G Mei $\beta$ ner, Nucl. Phys. B357 (1991) 90.

[15] G. Colangelo, M. Finkemeier and R. Urech, Phys. Rev. D54 (1996) 4403. 
[16] M.A. Samuel, J. Ellis and M. Karliner, Phys. Rev. Lett. 74 (1995) 4380;

J. Ellis, E. Gardi, M. Karliner and M. A. Samuel, Phys. Lett. B 366 (1996) 268.

[17] For a recent discussion on the inverse amplitude see: T. Hannah, Phys. Rev. D52 (1995) 4971; A. Dobado and J. R. Pelaez, "The Inverse Amplitude in Chiral Perturbation Theory", LBL preprint LBL-38645.

[18] K. Kawarabayashi and M. Suzuki, Phys. Rev. Lett. 16 (1966) 255; Riazuddin and Fayyazuddin, Phys. Rev. 147 (1966) 1071.

[19] L. Beldjoudi and T. N. Truong, Phys. Lett.B 351 (1995) 357.

[20] L. Beldjoudi and T. N. Truong, Talk Given at the International Symposium on Heavy Flavour and Electrweak Theory, Beijing, 1995.

[21] K. M. Watson, Phys. Rev. 95 (1955) 228.

[22] L. Beldjoudi and T. N. Truong, "Report No. CPTH A292.0294 (unpublished); L. Beldjoudi, Ph.D Thesis (1995) (unpublished)

[23] H. A. Bethe, Phys. Rev. 76 (1949) 38.

[24] S. Weinberg, Phys. Rev. Lett. 17 (1966) 616.

[25] L. S. Brown and R. L. Goble, Phys. Rev. Lett. 20 (1968) 346.

[26] P. Q. Hung, T. N. Pham and T. N. Truong, Phys. Rev. Lett. 59 (1987) 2251.

[27] Costa de Beauregard, T.N. Pham, B. Pire and T. N. Truong, Phys. Lett. B 67 (1977) 213.

[28] T. N. Truong (to be published).

[29] T. N. , Phys. Rev. D 30, 1509 (1984)

[30] G. J. Gounaris and J. J. Sakurai, Phys. Rev. Lett. 21 (1968) 24.

[31] S. R. Amendiola et al., Phys. Lett. B 146 (1984) 116; Phys. Lett. B 178 (1986) 435; Nucl. Phys. B 277 (1988) 168.

[32] J. F. Donoghue, J. Gasser and H. Leutwyler Nucl. Phys. B 340 (1990) 341.

[33] M. Boglione and M. R. Pennington, Univ. of Durham DTP-96/60 Jul, 1996 preprint.

[34] M. Ademollo and R. Gatto, Phys. Rev. Lett. 13 (1964) 264.

[35] "Decays of Tau Leptons to Final States Containing KS Mesons". (CLEO Collaboration) Preprint CLNS 96/1391, CLEO 96/3.

[36] L. Montanet etal. (Particle Data Group), Phys. Rev. D 50 (1994), and 1995. off-year partial update for the 1996 edition (URL:http://pdg.lbl.gov/).

[37] D. Morgan and M. R. Pennington, The Second DAPHNE Handbook, INFN - Laboratori Nazionali Di Fracasti, May 1995. 